\title{
Entre déterritorialisation et intersectionnalité, le « langage des bonnes» en littérature
}

\author{
Nella Arambašin \\ Français \\ nella.arambasin@univ-fcomte.fr
}

Dominées, les «subalternes ne peuvent pas parler », à moins d'analyser le « langage des bonnes » en littérature ; de la déterritorialisation à l'intersectionnalité, Kafka, Erri de Luca, Brassaï, Henri Miller, Fatou Diome et Milan Kundera permettent de saisir les résistances d'un langage genré, (dé)classé et racialisé.

Mots clé: langage de bonnes, déterritorialisation, intersectionnalité, migration, études subalternes.

Comme l'ont constaté Deleuze et Guattari en 1975, les interrogations sur ce qu'est une littérature " marginale » ou " populaire » se heurtent à des critères difficiles à fixer tant que le sujet de cette littérature est tenu pour l'effet d'un énoncé ou la cause de l'énonciation : en fait, il n'y aurait pas de sujet mais des " agencements collectifs d'énonciation », qui instaurent « du dedans un exercice mineur d'une langue même majeure » (32-34), usage qui caractérise la littérature dans un contexte sociopolitique de domination culturelle. Que Kafka puisse en être l'écrivain type à l'époque de la subordination du tchèque ou du yiddish à l'allemand, langue officielle de l'Empire habsbourgeois, ne peut cacher d'autres types de subordination linguistique, tel

le langage des bonnes [qui] n'est ni signifiant ni musical, il est ce son né du silence, que Kafka recherche partout, et où l'énoncé fait déjà partie d'un agencement collectif, d'une plainte collective, sans sujet d'énonciation qui se cache ou qui déforme. Pure matière mouvante d'expression. D'où leur qualité de personnages mineurs, d'autant plus dociles à la création littéraire. (119)

D'autorité, les écrivains sont donc les « porte-paroles » de locutrices dont le langage n'est pas codifié (Bourdieu 1982 : 64-68). Pourtant, comme les patois dans le théâtre français d'Ancien Régime ou celui de la paysanne italienne Giuseppina au XXe siècle, ce langage relève d'un mode relationnel. Erri de Luca explique que la bonne illettrée et son fiancé analphabète communiquaient d'un bout à l'autre de l'Italie en passant par deux intermédiaires, pour s'écrire et s'entendre scander les syllabes de lettres d'amour dont nous n'entendrons jamais l'intonation, mais 
qui par ce «stratagème » vocal pluriel doublement filtré et transformé préservait leur intimité et la sonorité dialectale (2013 : 9-17). Ce plurilinguisme " intérieur » à la langue nationale opère " une relativisation profonde de la conscience littéraire et linguistique », car elle permet non seulement de ressentir la « forme interne » de son propre langage comme « étrangère », mais aussi de participer " organiquement à un univers d'éclairage mutuel des langages », aussi multiples que relatifs les uns aux autres (Bakhtine 1978 : 184).

Si ce langage des bonnes ne relevait pas du « phénomène social de l'interaction verbale » que le cercle de Bakhtine tient pour « la réalité fondamentale de la langue » $(1977: 136,34)$ - ce que les linguistes ont pu décrire comme des " sociolectes avant la lettre » en prenant précisément la littérature pour objet d'analyse (Grutman 2019) - , il n'aurait laissé nulle trace et serait resté lettre morte. Les écrivains décentralisent donc «le monde idéologique » avec une « conscience galiléenne du langage » (Bakhtine 1978 : 183), à laquelle participe la mobilité sociolinguistique d'une main d'oeuvre pauvre, féminine, périphérique. En faire l'analyse d'un point de vue intersectionnel (de classe-genre-race), « permet d'éprouver, de diagnostiquer, les épistémologies de la domination comme les stratégies de résistances qui en découlent » (Dorlin 2008 : 83).

Les femmes occupent massivement les fonctions de services à la personne et d'entretien du foyer, une caractéristique de genre non questionnée par Kafka, remarque Nancy Huston ${ }^{1}$. En tant qu'ombres négligeables de la réalité quotidienne, elles sont destinées à projeter des fantasmes dans l'espace romanesque ; seul leur corps fait signe. Réduites à « des personnages silencieux et subordonnés [qui] font tout ce qu'on suppose qu'ils vont faire » dit Kafka dans son Journal (1973 : 379), elles ne peuvent à l'évidence pas parler («the subalterne cannot speak » : Spivak 1988). Plus encore, l'idiome patriarcal dominant présuppose leur pensée, puisqu'en tournant dans le vase clos et privé de la domesticité, elles se conforment aux échanges sexuels attendus d'elles ; tributaires du stéréotype multiséculaire de la servante-putain, « elles sont anti-conjugales et anti-familiales » (Deleuze/ Guattari 1975 :117). Pourtant, chacune possède une manière de « marquer » son territoire - «'façon de poivre' de Leni, odeur de la maison d'Olga : les restes de devenirs animaux » (124) - une aesthesis du corps-terroir qui incarne un «dedans » de la langue majeure, son « point de sous-développement, son propre patois, son tiers monde à soi » (33-34), à partir duquel l'écrivain « s'enfonce avec elles sur une ligne de déterritorialisation » (119). Le langage des bonnes balbutie dans ce patois tiers-mondiste kafkaïen : il touche aux structures patriarcales de l'enfermement domestique.

D'où la nécessité de compléter l'analyse par la notion de « rapports sociaux de sexe » (Dorlin 2008 :15-17), qu'emploient les féministes françaises des années

1 «Que la 'tour d'ivoire' ait parfois été une cave n'y change rien : 'j'ai souvent pensé, écrit Kafka, que la meilleure façon de vivre pour moi, serait de m'installer avec une lampe et ce qu'il faut pour écrire au coeur d'une vaste cave isolée. On m'apporterait les repas...' Je l'arrête là. » (Huston 2001: 295) 
1980 pour distinguer la division entre travail reproductif et productif. Que l'activité domestique puisse ainsi sortir de son inexistence académique, engage "l'ouverture conceptuelle à la figure de la femme immigrée ", dont la réalité longtemps sous-estimée par les représentations sociales était maintenue à l'ombre des activités productrices des hommes - les femmes employées aux «travaux précaires de l'économie informelle » étant effacées par un " cumul d'invisibilités », à la fois ethnique, statistique, politique, socio-économique et scientifique (Oso Casas 2001: 75, 58-59). Cette ouverture conceptuelle interroge à son tour la manière dont la littérature peut identifier, à rebours de l'expansion remarquable des échanges internationaux, l'invisibilité des chemins de la migration domestique.

Ne plus penser « l'émigration au masculin » engage Erri de Luca à se tourner vers les étrangères, prostituées ou servantes qu'il qualifie pourtant de « saintes » du judaïsme biblique ; emblématique, Giuseppina les introduit et sa sainteté s'étend aux anonymes « filles de la campagne [qui] quittaient les villages pour aller se placer dans les villes », où déracinées, elles devenaient « hôte dans la maison des autres, dans une chambre de bonne et dans une langue d'autres personnes. Là, on parlait un italien sans accent régional. » (De Luca 2013 : 8-9). Etrangère à la maison qu'elle habite, la bonne introduit « dans le langage autorisé la 'voix du peuple' », dont Certeau ne donne pas de définition, si ce n'est qu'il tient pour une « parole sauvage, religieuse, folle, enfantine ou populaire » celle qui traverse « la maison du langage comme des étrangères, en 'folles du logis' » (1980 : 232). Dans le logis du logos cette voix de l'imaginaire du peuple se signale par son étrangeté, "'quelque chose' d'autre parle encore » et « échappe » aux normes (230).

Pour un expatrié à Paris dans les années 1920, raconter l'Histoire de Marie c'est mettre en mots la résistance de cette "folle du logis », une femme de ménage étrangère que la société entière - avec homme d'affaires, huissier, avocat, propriétaire et locataires - tente d'expulser de sa chambre du huitième étage. Le hongrois Brassaï, photographe qui à l'époque écrivait et peignait, conduit Marie à sauver son taudis avec un français dont l'incorrection provient moins d'une autre langue, que d'autres compréhensions des unités sémantiques, syntaxiques et sonorités françaises ${ }^{2}$. Les effets de sens obtenus à partir des expressions idiomatiques deviennent doublement figurés, un burlesque poétique signifié par le choix du nom de famille de Marie : Malarmé. Avec les images tirées des expressions entendues, cadrées dans de courtes saynètes dramaturgiques, Brassaï compose un « curieux portrait-tableau » - celui « d'un nobody » dit Henry Miller (1995 : 11-12). Expatrié aussi, l'ami romancier américain se reconnaît dans ce portrait de l'artiste en femme de ménage, car ce « quelque chose qui s'épanouit dans notre for intérieur » lui rappelle le « triste paria que nous aurons toujours avec nous »:

2 «Y a un coussi-cuit dedans [le Chauffage]» (1995:22) ; « Ils étaient même alliancés! »(29), «A l'âge que je suis rendue » (35 et 57), «c'est pas une Rente visagère » (39), «Tout le monde veut l'en-ci, l'en-ça » (56), « ça me dit rien qui vaille bon » (62), « Il était en Convivance avec la concierge » (65), « aller au Ouatère » (80), « elle m'a insoufflé la Chance. C'est pas un conte de fou !» (69), «j'peux pas bien détourner mes mots » (83), «c'est pas la peine de cracher ces choses sur les toits » (83). 
Les choses qui lui arrivent, ses réactions, ses réflexions, son langage même, nous les connaissons intimement et nous les reconnaissons comme si Marie était, - ce qu'elle est réellement, une part de nous-mêmes. Son type, probablement, n’a pas beaucoup changé depuis l'époque sumérienne.[...] Les Maries de ce monde, toutes «mal-armées », savent qu'elles sont condamnées, que sous aucun régime, on ne fera rien pour les soulager. Elles récitent à haute voix leur complainte et cela résonne comme si elles se parlaient à ellesmêmes. [...] Brassaï a saisi tous ces pathétiques récitatifs pour les couler dans une série d'images éloquentes, dans une sorte de documentaire [...] (12-13)

De la femme anonyme réelle à sa représentation sumérienne ou biblique, la vie d'une bonne s'écrit en passant d'une singularité documentée à un pluriel auquel appartient son « agencement collectif d'énonciation » et qui amplifie le solo plaintif, depuis sa source immémoriale, en l'expression d'une revendication. Mais entendre et écrire s'articulent de manière ambivalente, comme deux niveaux de réalité difficilement compatibles, d'où découle un humour mêlé d'impuissance - le pathétique d'une lutte inégale. Localisée, la complainte ne devient audible cependant qu'à l'occasion d'un dé-placement ou exil de la voix, que la migrante anonyme tente d'aménager en un nouvel espace de vie. Rendre habitable sa langue dans celle d'autrui ne relève pas d'une adaptation mais implique plutôt ce que la sociolinguiste Caroline Panis appelle un « processus de géographisation linguistique », nécessitant « de mettre l'espace en narration » dans sa relation à des «frontières » (Canut et alii 2018 : 220, 228 / Panis : 231). Entre affrontements sociopolitiques et décalages poétiques des frontières, la domestication du logis est travaillée par sa déterritorialisation, une résistance conflictuelle qui touche chez les écrivains une dimension aussi intime que l'émigration intérieure de leur langue et qui les pousse loin des assignations d'une identité de genre.

Sur cette frontière entre histoire sociale et littérature, la réalité ethnolinguistique du langage des bonnes requiert une visibilité, sous-tendue par la racialisation des rapports sociaux de sexe. L'anthropologue James Scott en donne un modèle d'analyse quand il nomme « texte public, visible sur scène », celui que conteste en coulisses le «texte caché » ayant échappé à la " surveillance » du régime (2008 : 144). L'écrivaine Fatou Diome, qui choisit de rendre carnavalesque le texte public, utilise le stéréotype racial pour que l'idiotie de la parole dominante devienne manifeste et que soit inversé le stigmate. Dans son recueil de nouvelles La préférence nationale, elle endosse le rôle convenu de la bonne sénégalaise à laquelle on s'adresse d'emblée en « petit nègre » (2000:77). Faisant rimer « noir avec ignare »(70), la patronne française se décivilise en français, alors que l'émigrée africaine détient une licence de Lettres, son texte caché (76).

Autrement scénique est la mise en échec du consentement à la domination chez Milan Kundera. Son roman, La fête de l'insignifiance, décrit une réception mondaine à Paris, texte visible d'un rituel social qui justifie et codifie la hiérarchie des contacts publics, derrière lequel se tient une domesticité qui ne suscite « aucune attention », voire une « indifférence blasée » (2014 :70). Pourtant, la bonne portugaise est sommée par sa patronne de ne pas se montrer "une seule seconde au salon », car si les invités la «voyaient, ils s'enfuiraient »: 
l'injonction à l'invisibilité publique est argumentée par le stade onomatopéique d'une humanité animale, à savoir «l'air d'un oiseau d'Afrique : d'un perroquet de Bourenbouboubou », auquel l'ajout d'un rouge à lèvre donne l'implicite couleur de peau (72). En parallèle, le prénom Caliban que se choisit un acteur français au chômage, serveur à cette même réception, introduit une référence shakespearienne à l'esclavage, qu'une veste blanche orientalise en le rendant « exotique » (Idem). Clairement, l'existence sociale des Noir.e.s demeure genrée et connotée par l'histoire coloniale, mais pour la désigner il faut en passer par des stéréotypes visuels que le langage euphémise. En réaction, Kundera ne réduit pas le langage des subalternes à un français de cuisine mais en orchestre la forme clandestine ; Caliban, ayant décidé de jouer le rôle d'un Pakistanais non francophone, s'invente un parler crédible (69), une farce sonore laborieuse vite dégradée en gesticulations primaires par les invités, mais qui se mutualise au contact du portugais en coulisses :

Puisque Caliban lui parlait en pakistanais, elle avait une rare occasion de laisser tomber le français, langue qu'elle n'aimait pas, et de ne se servir, elle aussi, que de sa langue natale. Leur communication dans deux langues qu'ils ne comprenaient pas les rendit proches l'un de l'autre. (71)

Ponctué de parenthèses métalinguistiques, ce texte caché signale en français que les personnages se parlent alternativement en portugais ou en pseudopakistanais. Aucun idiome étranger n'apparaît, alors que le roman translingue (écrit en français par un auteur d'origine tchèque) a d'abord été traduit et publié en italien. Constitué de strates invisibles où circulent les langues étrangères avec leur imaginaire (post)colonial, l'usage public du français est régi par des rapports de race-classe-genre. La déterritorialisation du français ne passe pas par la fuite des Parisiens de leur salon où aurait surgi un étrange volatile, ni par une sexuation qui transformerait la servante portugaise en fantasme ancillaire. Une déterritorialisation traverse les frontières de l'incommunicabilité pour s'ouvrir à une intimité partagée, « pleine de bonté » et de " pudeur » aux couleurs « nostalgiques » $(109,110)$. Le langage des subalternes ne fait rire personne, il annonce « le crépuscule des plaisanteries! L'époque de l'après-blague » (96), murmure qui rend audible les migrantes hantées par la nostalgie de leur langue, comme l'est sans doute aussi Kundera.

\section{Bibliographie}

Bakhtine, Mikhaël / Volochinov, Valentin (1977). Le marxisme et la philosophie du langage. Essai de la méthode sociologique en linguistique, Paris : Ed. Minuit. [1 ère ed. 1929].

Bakhtine, Mikhaël (1978). Esthétique et théorie du roman, Paris : Gallimard.

Bourdieu, Pierre (1982). Ce que parler veut dire. L'économie des échanges symboliques, Paris : Fayard. 
Brassaï (1995). Histoire de Marie (1948), Paris : Actes Sud. Introduction de Henry Miller, pp. 9-15.

Canut, Cécile/ Danos, Félix/ Him-Aquilli, Manon/ Panis, Caroline (2018). La langage, une pratique sociale. Eléments d'une sociolinguistique politique, Besançon : PUFC. Partie 6 (Espaces et historicité).

Certeau, Michel de (1980). L'invention du quotidien, Paris : Gallimard, t.1. Récits d'espace, pp.170-191.

Deleuze, Gilles/ Guattari, Félix (1975). Kafka : pour une littérature mineure, Paris : Ed. Minuit.

De Luca, Erri (2013). Les saintes du scandale, Paris : Mercure de France. « Histoire de Giuseppina » pp.7-14. Traduit de l'italien par Danièle Valin, Le Sante dello scandalo, 2011.

Dorlin, Elsa (2008). Sexe, genre et sexualités : introduction à la théorie féministe, Paris : PUF.

Grutman, Rainer (1919). Sociolecte, in : Le lexique socius. Ressources sur le littéraire et le social.<http://ressources-socius.info/index.php/lexique/21-lexique/49sociolecte> (21/08/2019).

Kundera, Milan (2014). La Fête de l'insignifiance, Paris : Gallimard. [1 ${ }^{\text {ère }}$ ed. La Festa dell'insignificanza, 2013].

Huston, Nancy (2001). Le journal de la création, Paris : Babel. [1 ${ }^{\mathrm{ère}} \mathrm{ed.}$ 1990].

Kafka, Franz (1973). Journal, Paris : Grasset.

Oso Casas, Laura (2001). Domestiques, concierges et prostituées : migration et mobilité sociale des femmes immigrées, espagnoles à Paris, équatoriennes et colombiennes en Espagne (thèse Paris 1).

Scott, James C. (2008). La domination et les arts de la résistance : fragments du discours subalterne, Paris : Amsterdam Ed. [1 $1^{\text {ère }}$ ed. 1992].

Spivak, Gayatri C. (1988). Can the Subaltern Speak? In : Marxim and the Interpretation of Culture, N. Nelson/ L. Crossberg Ed., Chicago : University of Illinois Press, pp.271-313.

\section{Između deteritorializacije $\mathrm{i}$ interseksionalizma, « Jezik sluškinja » u literaturi}

Iako se zna da « subalterne žene ne mogu govoriti », valja analizirati «jezik sluškinja » u literaturi kao otpor dominantnom govoru ; od deteritorializacije nacionalnih jezika do interseksionalizma, Kafka, Erri de Luca, Brassaï, Henri Miller, Fatou Diome i Milan Kundera omogućuju shvatiti stvaranje strategija jednog jezika - rodno, klasno i rasno determiniran.

Kljucne rijeci : jezik sluškinje, deteritorializacija, interseksionalizam, migracije, subalterne studije. 